PROCEEDINGS OF THE

AMERICAN MATHEMATICAL SOCIETY

Volume 138, Number 12, December 2010, Pages 4455-4468

S 0002-9939(2010)10499-9

Article electronically published on July 19, 2010

\title{
ON ROBUSTNESS OF EXACT CONTROLLABILITY AND EXACT OBSERVABILITY UNDER CROSS PERTURBATIONS OF THE GENERATOR IN BANACH SPACES
}

\author{
ZHAN-DONG MEI AND JI-GEN PENG
}

(Communicated by Nigel J. Kalton)

\begin{abstract}
This paper is concerned with the exact controllability and exact observability of linear systems in the Banach space setting. It is proved that both the admissibility of control operators and the admissibility of observation operators are invariant to cross perturbations of the generator of a $C_{0}$-semigroup. Moreover, under the admissibility invariance premise, the robustness of the exact controllability as well as the exact observability to such cross perturbations is verified. An illustrative example is presented.
\end{abstract}

\section{INTRODUCTION}

Consider the infinite dimensional linear systems described by the following differential equations:

$$
\left\{\begin{array}{l}
\dot{x}(t)=A x(t)+B u(t), t \geq 0, \\
y(t)=C x(t), \\
x(0)=x_{0},
\end{array}\right.
$$

where the system state $x(t)$ takes values in Banach space $X$; the coefficient operator $A: D(A) \subset X \rightarrow X$ generates a $C_{0}$-semigroup $\{T(t)\}_{t \geq 0}$; the input $u(\cdot)$ and output $y(\cdot)$ take values in Banach spaces $U$ and $Y$, respectively; the control operator $B$ is bounded from $U$ to the extrapolation space $X_{-1}^{A}$ of $X$; and the observation operator $C$ is bounded from $X_{1}^{A}$ to $Y$, where $X_{1}^{A}$ denotes the domain $D(A)$ equipped with the graph norm. By [3], the extrapolation space $X_{-1}^{A}$ is the completion of $X$ under the norm $\left\|R\left(\lambda_{0}, A\right) \cdot\right\|$, with $R\left(\lambda_{0}, A\right)$ the resolvent of $A$ at $\lambda_{0}$.

Let $\left\{T_{-1}(t)\right\}_{t \geq 0}$ be the extrapolation semigroup of $\{T(t)\}_{t \geq 0}$ on $X_{-1}^{A}$. In order to guarantee that the state $x(t)=T(t) x_{0}+\int_{0}^{t} T_{-1}(t-s) B u(s) d s$ stays in $X$ for any $x_{0} \in X, u \in L^{p}([0, \infty), U)(p \in(1, \infty))$ and $t>0$, we introduce the notion of an admissible control operator. The control operator $B$ is said to be admissible for $A$ if, for any $t \geq 0$, the mapping $M(t): u \mapsto \int_{0}^{t} T_{-1}(t-s) B u(s) d s$ is continuous from $L^{p}([0, \infty), U)$ into $X$. If, in addition, $U=X$, we say $B$ is an admissible control

Received by the editors November 27, 2009 and, in revised form, February 4, 2010 and March 12, 2010.

2010 Mathematics Subject Classification. Primary 93C25, 93B05, 93B07.

Key words and phrases. $C_{0}$-semigroup, cross perturbation, admissibility, robustness, exact controllability, exact observability.

This work was supported by the Natural Science Foundation of China under contract No. 60970149.

(C) 2010 American Mathematical Society 4455

Reverts to public domain 28 years from publication 
perturbation for $A$. For the free system (1.1) $(u=0)$, in order to guarantee the output $y \in L^{p}([0, \infty), Y)$, we introduce the notion of an admissible observation operator. The observation operator $C$ is said to be admissible for $A$ if for some $T>0$ there exists a constant $K>0$ such that

$$
\int_{0}^{T}\|C T(t) x\|_{Y}^{p} d t \leq K\|x\|_{X}^{p}, \forall x \in D(A) .
$$

If in addition, $Y=X$, we say $C$ is an admissible observation perturbation for A. Admissible control and admissible observation are closely related to infinite dimensional linear systems with boundary control and point observation (see 21, 25, 26]). It is proved in [26] that there are some dual relationships of admissible control and admissible observation in the case that $X$ is reflexive and $1<p<\infty$.

The pair $(A, B)$ with $B$ being admissible for $A$ is said to be exactly controllable at $\tau>0$ if $\Phi_{A, B}(\tau)$ (see Section 2) is surjective. The pair $(A, C)$ with $C$ being admissible for $A$ is said to be exactly observable at $\tau>0$ if there exists a constant $k>0$ such that $\left\|\Psi_{A, C}(\tau) x\right\| \geq k\|x\|$ for any $x \in X$ (see Section 2 for $\Psi_{A, C}$ ). Exact controllability and exact observability are extensions of controllability and observability from finite dimensional systems theory to infinite dimensions. It is well known that controllability enters into the study of many other important concepts such as stabilizability and optimizability; observability enters into the study of many other important concepts such as detectability and estimatability. In the Hilbert space setting and in the particular case that $p=2$, it is proved in [24] that exact controllability and exact observability are dual to each other. That is, the exact controllability of $(A, B)$ is equivalent to the exact observability of $\left(A^{*}, B^{*}\right)$ at the same time $\tau$, where $A^{*}$ and $B^{*}$ are the corresponding dual operators. Such duality was first formulated in Dolecki and Russell [2, but it was used for proving the exact controllability of PDE's systems only several years later; see e.g. Lions [15, 16] and Triggiani 23. Exact controllability and exact observability have received considerable attention in the functional analysis frame (see e.g. [4, 10, 18, 19, 20, 30), where some necessary and/or sufficient conditions have been given.

There are many references that focus on the robustness of exact controllability and exact observability (namely, the persistence of exact controllability and exact observability under some small perturbations). In the finite dimensional space setting, Lee and Markus [13 have proved that if $(A, B)$ is (exactly) controllable, then there exists an $\epsilon>0$ such that for all $\|\Delta A\|<\epsilon$ and $\|\Delta B\|<\epsilon$, where $\Delta A$ and $\Delta B$ are matrices of appropriate dimensions, the linear system $\dot{x}(t)=$ $(A+\Delta A) x(t)+(B+\Delta B) u(t), t \geq 0$, remains (exactly) controllable. This means that the controllability of $(A, B)$ is not affected by "small" perturbations. Since the system is of finite dimensions, the (exact) controllability is also robust by the dual relationship.

In the infinite dimensional space setting, Leiva [14 has considered some class of unbounded perturbations $P: D(A) \rightarrow X$ which is not too irregular with respect to $A$. He proved that if the system $\dot{x}(t)=A x(t)+B(t) u(t)$, with $B(\cdot)$ being bounded and continuous in the strong operator topology of the space of all the bounded linear operators from $U$ to $X$, is exactly controllable, then it is as well for all systems $\dot{x}(t)=A x(t)+P x(t)+B(t) u(t)$ with $P$ near to 0 (w.r.t. some metric). The authors of 1 proved that for bounded control operators in $X$, the exact controllability of $\dot{x}(t)=A x(t)+B(t) u(t)$ implies that of the perturbed system $\dot{x}(t)=A x(t)+\Delta A x(t)+B(t) u(t)$ for $\Delta A$ belonging to a neighborhood of zero in the 
class of Desch-Schappacher perturbations. The arguments used in [1] relies heavily on estimates using Dyson-Phillips series, involving calculations facilitated only by the boundedness of the control operator on $X$.

However, in partial differential equations the control usually acts on the boundary. Such systems can be reformulated as distributed control systems of the form $\dot{x}(t)=A x(t)+B u(t)$, where the control operator is strictly unbounded (see [17, 21]). These cases are beyond the scope of [1] and [14 which considered the case that the control operator $B$ is bounded. Dual to control, the observation is usually of point evaluation, and thereby unbounded. Therefore, it is significant to consider exact controllability and exact observability in the case that the control operator $B$ and/or the observation operator $C$ are unbounded but admissible. It was proved in 29] that for a well-posed linear system with admissible feedback operator the closed-loop system inherits the exact controllability and exact observability of the open-loop system. Furthermore, it was proved that there exists an admissible feedback radius $r_{0}$, such that any bounded linear operator $K \in L(Y, U)$ satisfying $\|K\|<r_{0}$ is an admissible feedback operator for the open-loop system. Hence the closed-loop system keeps the exact observability and exact controllability under the small perturbation of admissible feedback. In this sense, admissible feedback can be regarded as a robust perturbation of exact controllability and exact observability of system (1.1).

Recently, Hadd proved in [7] the admissibility invariance of the control operator to the admissible control perturbation of the generator, and under this premise he proved the robustness of exact controllability. Moreover, he proved in [6, 8] the invariance of admissible observability under admissible observation perturbation. The robustness of exact observability under admissible observation perturbation has been verified by Tucsnak and Weiss [24, Theorem 6.3.2, page 186]. It is easy to see that [6] and 24] are concerned with the admissible control perturbation to exact controllability and the admissible observation perturbation to exact observability, respectively. To the best of the authors' knowledge, for unbounded generator, control and observation operators, there is no literature which discusses the cross perturbations, that is, admissible control perturbation to exact observability and admissible observation perturbation to exact controllability (in fact, the work of [14] can be regarded as cross perturbation for the bounded control operator).

This paper is devoted to investigating in the Banach space setting the robustness of exact controllability and exact observability under cross perturbations; that is, if $(A, B)((A, C))$ is exactly controllable (exactly observable), then $(A+P, B)\left(\left(\left(A_{-1}+\right.\right.\right.$ $\left.\Delta A)\left.\right|_{X}, C_{\Lambda}^{A}\right)$ ) (see Section 2 for $C_{\Lambda}^{A}$ ) is exactly controllable (exactly observable) whenever $P(\Delta A)$ is small under some metric, where $\left.\left(A_{-1}+\Delta A\right)\right|_{X}$ is the part of $A+\Delta A$ in $X$ defined by $\left.\left(A_{-1}+\Delta A\right)\right|_{X} x=A_{-1} x+\Delta A x$ with the domain $D\left(\left.\left(A_{-1}+\Delta A\right)\right|_{X}\right)=\left\{x \in X:\left(A_{-1}+\Delta A\right) x \in X\right\}$. It should be noted that in the Banach space setting, exact controllability and exact observability are not exactly dual to each other. In fact, the admissibility of $B$ for $A$ and $B^{*}$ for $A^{*}$ need not be equivalent because in a general Banach space even $A^{*}$ need not be a generator of a $C_{0}$-semigroup. Furthermore, there is no dual form of $\Lambda$-extension (see [26]). Hence, the robustness of controllability and observability can't be obtained by the duality from one to the other, and so they are both worth considering. Our procedure is as follows. In Section 2, we give some necessary background on regular linear system, which is the main tool of our paper. In Section 3, we 
first prove the invariance of admissible controllability and admissible observability under cross perturbations with regularity assumptions, and under such admissibility invariance premise, we prove the robustness of exact controllability and that of exact observability, respectively. Finally, an illustrative example is given.

Throughout this paper, $X, U$ and $Y$ are Banach spaces, $(A, D(A))$ is the generator of the $C_{0}$-semigroup $T:=(T(t))_{t \geq 0}$ on $X$, and $1<p<\infty$. We denote by $L(U, X)$ all the bounded linear operators from $U$ to $X$ and by $\left.M\right|_{X}=\{x \in D(M)$ : $M x \in X\}$ the part of $M$ in $X$. Denote by $I_{X}$ the identity operator on $X$ and denote $R^{+}=[0, \infty)$. For $u, v \in L^{p}\left(R^{+}, U\right)$ and $\tau \geq 0$, the $\tau$-concatenation of $u$ and $v$, denoted by $u \underset{\tau}{\diamond} v$, is defined by

$$
\left(u \diamond_{\tau} v\right)(t)= \begin{cases}u(t), & t<\tau \\ v(t-\tau), & t \geq \tau .\end{cases}
$$

\section{BACKGROUND ON REGULAR LINEAR SYSTEMS}

This section is to recall in a very sketchy way the concept of the regular linear system which is to be used as the main tool in the next section in the sense of Salamon [21] and Weiss 28].

The pair $(T, \Phi):=\left((T(t))_{t \geq 0},(\Phi(t))_{t \geq 0}\right)$ is called an abstract linear control system on $(X, U)$ if $\{\Phi(t)\}_{t \geq 0}$ is a one-parameter family of linear bounded operators from $L^{p}\left(R^{+}, U\right)$ to $X$ satisfying

$$
\Phi(t+\tau)(u \underset{\tau}{\diamond} v)=T(t) \Phi(\tau) u+\Phi(t) v, \forall u, v \in L^{p}\left(R^{+}, U\right), \forall t, \tau \geq 0 .
$$

By the representation theorem due to Weiss [25, there exists a unique operator $B \in L\left(U, X_{-1}\right)$, which is admissible for $A$, such that for any $t \geq 0$ and $u \in$ $L^{p}\left(R^{+}, U\right)$,

$$
\Phi(t) u=\int_{0}^{t} T_{-1}(t-s) B u(s) d s \in X
$$

where the integral exists in $X_{-1}$. Conversely, for any admissible control operator $B \in L\left(U, X_{-1}\right)$ there corresponds a unique control system $(T, \Phi)$. So it is reasonable to denote $\Phi=\Phi_{A, B}$.

The pair $(T, \Psi)=\left((T(t))_{t \geq 0},(\Psi(t))_{t \geq 0}\right)$ is called an abstract linear observation system on $(X, Y)$ if $\{\Psi(t)\}_{t \geq 0}$ is a one-parameter family of bounded linear operators from $X$ to $L^{p}\left(R^{+}, Y\right)$ that satisfies

$$
\Psi(t+\tau) x=\Psi(\tau) x \underset{\tau}{\diamond} \Psi(t) T(\tau) x, \forall t, \tau>0, x \in X .
$$

By the representation theorem in [26], there exists a unique operator $C \in$ $L\left(X_{1}, Y\right)$, which is admissible for $A$, such that for any $t \geq 0$ and $x \in D(A)$, $C T(t) x=(\Psi(\infty) x)(t)$, where $\Psi(\infty)$, which is continuous from $X$ to $L_{l o c}^{p}\left(R^{+}, Y\right)$, is the extended output map defined by the strong limit of $\Psi(\tau)$ as $\tau \rightarrow \infty$ (see [26]). Conversely, for any admissible observe operator $C \in L\left(X_{1}, Y\right)$ there corresponds a unique abstract linear observation system $(T, \Psi)$. So it is reasonable to denote $\Psi=\Psi_{A, C}$.

Assume $T, \Phi$ and $\Psi$ are as above. The quadruple $\Sigma=(T, \Phi, \Psi, F)$ is said to be a well-posed linear system on $(X, U, Y)$ if, in addition, $F=(F(t))_{t \geq 0}$ is a family of bounded linear operators from $L^{p}\left(R^{+}, U\right)$ to $L^{p}\left(R^{+}, Y\right)$ satisfying

$$
F(t+\tau)(u \underset{\tau}{\diamond v})=F(\tau) u \underset{\tau}{\diamond}(\Psi(t) \Phi(\tau) u+F(t) v), \forall u, v \in L^{p}\left(R^{+}, U\right) .
$$


We say that the well-posed linear system $\Sigma$ is regular if the limit

$$
\lim _{t \rightarrow 0} \frac{1}{t} \int_{0}^{t}\left(F(\infty) u_{0}\right)(s) d s=D z
$$

exists in $Y$ for the constant input $u_{0}(t)=z$, where $F(\infty)$, which is continuous from $L_{l o c}^{p}\left(R^{+}, U\right)$ to $L_{l o c}^{p}\left(R^{+}, Y\right)$, is the extended input-output map defined by the strong limit of $F(\tau)$ as $\tau \rightarrow \infty$ (see [27]). Moreover, we call $D$ to be the feedthrough operator of $\Sigma$. In this case, we say that the regular linear system $\Sigma=(T, \Phi, \Psi, F)$ is generated by $(A, B, C, D)$, and we denote $\Sigma=\Sigma_{A, B, C, D}$. If $D=0$, we denote $F=F_{A, B, C},(A, B, C)=(A, B, C, 0)$ and $\Sigma_{A, B, C}=\Sigma_{A, B, C, 0}$ for convenience.

In order to introduce the representation theorem of the regular linear system, Weiss [28 introduced an extension of $C$, called a $\Lambda$-extension with respect to $A$, which is defined by

$$
C_{\Lambda}^{A} x=\lim _{\lambda \rightarrow \infty} C \lambda R(\lambda, A) x
$$

with the domain $D\left(C_{\Lambda}^{A}\right)=\{x \in X$ : this above limit exists in $Y\}$.

With the $\Lambda$-extension, for the well-posed linear system $\Sigma$, the regularity condition (2.5) is equivalent to each of the following two conditions:

a) Range $\left(R\left(\lambda, A_{-1}\right) B\right) \subset D\left(C_{\Lambda}^{A}\right)$ holds for some (and hence for all) $\lambda \in \rho(A)$.

b) For any $u \in U, G(\lambda) u$ has a limits when $\lambda \rightarrow \infty$, where $G$ is the transfer function associated to $F(\infty)$.

In this case, the transfer function $G=G_{A, B, C, D}$ is given explicitly by

$$
G(\lambda)=C_{\Lambda}^{A} R\left(\lambda, A_{-1}\right) B+D, \operatorname{Re}(\lambda)>w_{0}(T),
$$

where $w_{0}(T)$ is the growth bound of the $C_{0}$-semigroup $T$. We denote $G_{A, B, C}=$ $G_{A, B, C, 0}$ for short.

Next, we consider the feedback of the regular linear system; see [22] and [29] for details.

Definition 2.1. Let $\Sigma$ be a regular linear system on $(X, U, Y)$ with input-output operators $F(t)$. An operator $\Gamma \in L(Y, U)$ is called an admissible feedback operator for $\Sigma$ if $I-F(\cdot) \Gamma$ have uniformly bounded inverses.

Theorem 2.2. Let $(A, B, C)$ generate a regular linear system $\Sigma=(T, \Phi, \Psi, F)$ on $(X, U, Y)$ with an admissible feedback operator $\Gamma \in L(Y, U)$. Then the feedback system $\Sigma^{\Gamma}$ is also a regular linear system given by

$$
\Sigma^{\Gamma}=\left(\begin{array}{cc}
T^{\Gamma}(\cdot) & \Phi^{\Gamma}(\cdot) \\
\Psi^{\Gamma}(\cdot) & F^{\Gamma}(\cdot)
\end{array}\right)=\left(\begin{array}{cc}
T(\cdot)+\Phi(\cdot) \Gamma(I-F \Gamma)^{-1} \Psi & \Phi(\cdot)(I-\Gamma F(\cdot))^{-1} \\
(I-\Gamma F(\cdot))^{-1} \Psi & F(I-\Gamma F(\cdot))^{-1}
\end{array}\right)
$$

with the generating operators $\left(A^{\Gamma}, B^{\Gamma}, C^{\Gamma}\right)$ :

$$
A^{\Gamma}=\left.\left(A_{-1}+B \Gamma C_{\Lambda}^{A}\right)\right|_{X}, D\left(A^{\Gamma}\right):=\left\{z \in D\left(C_{\Lambda}^{A}\right):\left(A_{-1}+B \Gamma C_{\Lambda}^{A}\right) z \in X\right\}
$$

and $B^{\Gamma}=J^{A, A^{\Gamma}} B, C^{\Gamma}=C_{\Lambda}^{A}$ restricted to $D\left(A^{\Gamma}\right)$, where $J^{A, A^{\Gamma}}$ is defined by $J^{A, A^{\Gamma}} x=\lim _{\lambda \rightarrow \infty}\left(\lambda-A_{-1}\right)^{-1} x$ (in $\left.X_{-1}^{A^{\Gamma}}\right)$ with $D\left(J^{A, A^{\Gamma}}\right)=\left\{x \in X_{-1}^{A}\right.$ : the limit $\lim _{\lambda \rightarrow \infty}\left(\lambda-A_{-1}\right)^{-1} x$ exists $\}$. In addition, $D\left(C_{\Lambda}^{A}\right)=D\left(\left(C^{\Gamma}\right)_{\Lambda}^{A^{\Gamma}}\right)$.

Remark 2.3. Let $\beta \in \rho(A)$. According to [29], the mapping $J^{A, A^{\Gamma}}$ defined above is an isomorphism from $W$ to $W^{\Gamma}$, where $W=\left(\beta-A_{-1}\right) W_{1}$, with $W_{1}$ being the closure of $X_{1}^{A}$ in $C_{\Lambda}^{A}$, and where $W^{\Gamma}$ is defined in the same way. Accordingly, we 
always consider $B$ as $J^{A, A^{\Gamma}} B$ for short (for example, in Theorem 3.9 we say " $B$ is admissible for $A+P$ " instead of " $J{ }^{A, A+P} B$ is admissible for $A+P$ ").

\section{MAin RESUlts}

In this section, we prove the robustness of exact controllability and exact observability to cross perturbations, respectively. As in [7], we have to find an appropriate metric to measure the robustness. To this purpose, we first introduce two lemmas.

Lemma 3.1. Assume that $(A, C)$ generates an abstract linear observation system on $(X, Y)$. Let $w \in R, M \geq 1$ such that $\|T(t)\| \leq M e^{w t}$ for $t \geq 0$. Then, we have that

$$
\left\|\Psi_{A, C}(\tau)\right\| \leq k\left(\tau, \tau_{0}\right)\left\|\Psi_{A, C}\left(\tau_{0}\right)\right\|, \forall \tau>0
$$

where

$$
k\left(\tau, \tau_{0}\right)= \begin{cases}M e^{w \tau} \frac{e^{w \tau} 0}{e^{w \tau} 0-1}, & w>0 \\ M\left(1+\frac{\tau}{\tau_{0}}\right), & w=0 \\ \frac{M}{1-e^{w \tau} \tau_{0}}, & w<0\end{cases}
$$

Proof. Similarly to the proof of [26, Proposition 2.3], it is easy to show that

$$
\left\|\Psi_{A, C}\left(n \tau_{0}\right)\right\| \leq\left\|\Psi_{A, C}\left(\tau_{0}\right)\right\| M \sum_{k=0}^{n-1} e^{w k \tau_{0}}, \forall n \in \mathbb{N} .
$$

Let $\tau>0$. Without loss of generality, assume that $\tau \in\left((n-1) \tau_{0}, n \tau_{0}\right]$ for a certain $n \in \mathbb{N}$. Then, by the above inequality it follows that

$$
\left\|\Psi_{A, C}(\tau)\right\| \leq\left\|\Psi_{A, C}\left(n \tau_{0}\right)\right\| \leq \begin{cases}\left\|\Psi_{A, C}\left(\tau_{0}\right)\right\| M e^{w \tau} \frac{e^{w \tau_{0}}}{e^{w \tau_{0}}-1}, & w>0 \\ \left\|\Psi_{A, C}\left(\tau_{0}\right)\right\| M\left(1+\frac{\tau}{\tau_{0}}\right), & w=0 \\ \left\|\Psi_{A, C}\left(\tau_{0}\right)\right\| \frac{M}{1-e^{w \tau_{0}}}, & w<0\end{cases}
$$

This completes the proof.

Lemma 3.2. Assume that $(A, B, C)$ generates a regular linear system on $(X, U, Y)$. Let $\tau_{0}>0$ and let $w \in R, M \geq 1$ such that $\|T(t)\| \leq M e^{w t}$ for $t \geq 0$. Then, for any $\tau>0$ we have

$$
\left\|F_{A, B, C}(\tau)\right\| \leq k\left(\tau, \tau_{0}\right)\left(1+\left\|\Phi_{A, B}\left(\tau_{0}\right)\right\|\right)\|C\|_{\mathscr{R}_{A, B}\left(\tau_{0}\right)}
$$

and

$$
\left\|F_{A, B, C}(\tau)\right\| \leq k\left(\tau, \tau_{0}\right)\left(1+\left\|\Psi_{A, C}\left(\tau_{0}\right)\right\|\right)\|B\|_{\mathscr{W}_{A, C}\left(\tau_{0}\right)},
$$

where $\|C\|_{\mathscr{R}_{A, B}\left(\tau_{0}\right)}=\left\|\Psi_{A, C}\left(\tau_{0}\right)\right\|+\left\|F_{A, B, C}\left(\tau_{0}\right)\right\|,\|B\|_{\mathscr{W}_{A, C}\left(\tau_{0}\right)}=\left\|\Phi_{A, B}\left(\tau_{0}\right)\right\|+$ $\left\|F_{A, B, C}\left(\tau_{0}\right)\right\|$, and $k\left(\tau, \tau_{0}\right)$ is the same as in Lemma 3.1 .

Proof. Similarly to the proof of [27, Proposition 2.1], it can be proved that, $\forall n \in \mathbb{N}$,

$$
\left\|F_{A, B, C}\left(n \tau_{0}\right)\right\| \leq\left\|F_{A, B, C}\left(\tau_{0}\right)\right\|+\left\|\Psi_{A, C}\left(\tau_{0}\right)\right\|\left\|\Phi_{A, B}\left(\tau_{0}\right)\right\| M \sum_{l=1}^{n-1} e^{w(l-1) \tau_{0}} .
$$


Let $\tau \in\left((n-1) \tau_{0}, n \tau_{0}\right]$ for some $n \in \mathbb{N}$, w.l.g. Then, (1) in the case that $w>0$ we have

$$
\begin{aligned}
\left\|F_{A, B, C}(\tau)\right\| & \leq\left\|F_{A, B, C}\left(\tau_{0}\right)\right\|+\left\|\Psi_{A, C}\left(\tau_{0}\right)\right\|\left\|\Phi_{A, B}\left(\tau_{0}\right)\right\| M \frac{e^{w n \tau_{0}}}{e^{w \tau_{0}}-1} \\
& \leq\left\|F_{A, B, C}\left(\tau_{0}\right)\right\|+\left\|\Psi_{A, C}\left(\tau_{0}\right)\right\|\left\|\Phi_{A, B}\left(\tau_{0}\right)\right\| M e^{w \tau_{0}} \frac{e^{w \tau}}{e^{w \tau_{0}}-1} \\
& \leq\left(\left\|F_{A, B, C}\left(\tau_{0}\right)\right\|+\left\|\Psi_{A, C}\left(\tau_{0}\right)\right\|\left\|\Phi_{A, B}\left(\tau_{0}\right)\right\|\right) M e^{w \tau_{0}} \frac{e^{w \tau}}{e^{w \tau_{0}}-1} \\
& \leq\|C\|_{\mathscr{R}_{A, B}} M e^{w \tau_{0}}\left(1+\left\|\Phi_{A, B}\left(\tau_{0}\right)\right\|\right) \frac{e^{w \tau}}{e^{w \tau_{0}}-1} ;
\end{aligned}
$$

(2) in the case that $w=0$ we have

$$
\begin{aligned}
\left\|F_{A, B, C}(\tau)\right\| & \leq\left\|F_{A, B, C}\left(\tau_{0}\right)\right\|+\left\|\Psi_{A, C}\left(\tau_{0}\right)\right\|\left\|\Phi_{A, B}\left(\tau_{0}\right)\right\| M n \\
& \leq\left\|F_{A, B, C}\left(\tau_{0}\right)\right\|+\left\|\Psi_{A, C}\left(\tau_{0}\right)\right\|\left\|\Phi_{A, B}\left(\tau_{0}\right)\right\| M\left(1+\tau / \tau_{0}\right) \\
& \leq\left(\left\|F_{A, B, C}\left(\tau_{0}\right)\right\|+\left\|\Psi_{A, C}\left(\tau_{0}\right)\right\|\left\|\Phi_{A, B}\left(\tau_{0}\right)\right\|\right) M\left(1+\tau / \tau_{0}\right) \\
& \leq\|C\|_{\mathscr{R}_{A, B}}\left(1+\left\|\Phi_{A, B}\left(\tau_{0}\right)\right\|\right) M\left(1+\tau / \tau_{0}\right)
\end{aligned}
$$

and (3) in the case that $w<0$ we have

$$
\begin{aligned}
\left\|F_{A, B, C}(\tau)\right\| & \leq\left\|F_{A, B, C}\left(\tau_{0}\right)\right\|+\left\|\Psi_{A, C}\left(\tau_{0}\right)\right\|\left\|\Phi_{A, B}\left(\tau_{0}\right)\right\| M \frac{1}{1-e^{w \tau_{0}}} \\
& \leq\left(\left\|F_{A, B, C}\left(\tau_{0}\right)\right\|+\left\|\Psi_{A, C}\left(\tau_{0}\right)\right\|\left\|\Phi_{A, B}\left(\tau_{0}\right)\right\|\right) M \frac{1}{1-e^{w \tau_{0}}} \\
& \leq\|C\|_{\mathscr{R}_{A, B}}\left(1+\left\|\Phi_{A, B}\left(\tau_{0}\right)\right\|\right) M \frac{1}{1-e^{w \tau_{0}}} .
\end{aligned}
$$

The three cases cover all possibilities, so the inequality (3.2) is proved. The inequality (3.3) can be proved similarly. The proof is therefore completed.

With the above two lemmas, we can obtain the following completeness result of a linear space, which is important for us to introduce a metric to describe the robustness of exact observability.

Theorem 3.3. If $(A, B)$ generates an abstract linear control system on $(X, U)$, then

$$
\mathscr{R}_{A, B}=\left\{R \in L\left(X_{1}^{A}, Y\right):(A, B, R) \text { generates a regular linear system }\right\}
$$

is a Banach space endowed with the norm

$$
\|R\|_{\mathscr{R}_{A, B}\left(\tau_{0}\right)}:=\left\|\Psi_{A, R}\left(\tau_{0}\right)\right\|+\left\|F_{A, B, R}\left(\tau_{0}\right)\right\|,
$$

for any fixed $\tau_{0}>0$.

Proof. It follows from the above two lemmas that for any $\tau_{1}, \tau_{2}$,

$$
\|R\|_{\mathscr{R}_{A, B}\left(\tau_{1}\right)} \leq k\left(\tau_{1}, \tau_{2}\right)\left(2+\left\|\Phi_{A, B}\left(\tau_{2}\right)\right\|\right)\|R\|_{\mathscr{R}_{A, B}\left(\tau_{2}\right)}, \forall R \in \mathscr{R}_{A, B},
$$

which shows that $\left\{\|\cdot\|_{\mathscr{R}_{A, B}(\tau)}\right\}_{\tau>0}$ is an equivalent norm family. To complete the proof, it suffices to show that $\mathscr{R}_{A, B}$ is complete under some norm $\|R\|_{\mathscr{R}_{A, B}(\tau)}$. Similarly to the proof of [9. Proposition 1], it is easy to show that $\mathscr{R}_{A, B}$ is a Banach space under the norm $\|R\|_{\mathscr{R}_{A, B}(1)}$. 
In order to derive the "dual" conclusion, we introduce the following lemma due to Said Hadd [7:

Lemma 3.4. Assume that $(A, B)$ generates an abstract linear control system on $(X, U)$. Let $(T(t))_{t \geq 0}$ be the $C_{0}$-semigroup generated by $A$ satisfying $\|T(t)\| \leq M e^{w t}$ for some $w \in R$. Then, we have

$$
\left\|\Phi_{A, B}(\tau)\right\| \leq k\left(\tau, \tau_{0}\right)\left\|\Phi_{A, B}\left(\tau_{0}\right)\right\|, \tau, \tau_{0}>0,
$$

where $k\left(\tau, \tau_{0}\right)$ is defined as in Lemma 3.1 .

Theorem 3.5. If $(A, C)$ generates an abstract linear observation system on $(X, Y)$, then

$$
\mathscr{W}_{A, C}=\left\{W \in L\left(U, X_{-1}\right):(A, W, C) \text { generates a regular linear system }\right\}
$$

is a Banach space endowed with the norm

$$
\|W\|_{\mathscr{W}_{A, C}\left(\tau_{0}\right)}:=\left\|\Phi_{A, W}\left(\tau_{0}\right)\right\|+\left\|F_{A, W, C}\left(\tau_{0}\right)\right\|
$$

for any fixed $\tau_{0}>0$.

Proof. By Lemma 3.2 and Lemma 3.4, it follows that $\forall \tau, \tau_{0}>0$,

$$
\|R\|_{\mathscr{W}_{A, C}(\tau)} \leq k\left(\tau, \tau_{0}\right)\left(2+\left\|\Psi_{A, C}\left(\tau_{0}\right)\right\|\right)\|R\|_{\mathscr{W}_{A, C}\left(\tau_{0}\right)},
$$

which indicates that $\left\{\|\cdot\|_{\mathscr{W}_{A, C}(\tau)}\right\}_{\tau>0}$ is a family of equivalent norms. So, to conclude the theorem, it is sufficient to show that $\mathscr{W}_{A, C}$ is completed under some norm $\|\cdot\|_{\mathscr{W}_{A, C}(\tau)}$. In the same way as with the proof of [9, Proposition 1], we can show that $\mathscr{W}_{A, C}$ is a Banach space for the norm $\|R\|_{\mathscr{W}_{A, C}(1)}$, as expected.

The following famous lemma, which says that the subset of surjective operators is open in the space of bounded linear operators, gives an important tool to prove the robustness of exact controllability to cross perturbations. For convenience, we define the set

$$
\mathfrak{S}(E, F)=\{\Xi \in L(E, F): \Xi \text { is surjective }\},
$$

for Banach spaces $E$ and $F$.

Lemma 3.6 ([11, page 227]). Let $E$ and $F$ be Banach spaces. Then, $\mathfrak{S}(E, F)$ is an open set in $L(E, F)$; i.e., given $\Pi \in \mathfrak{S}(E, F)$, there exists $\alpha>0$ such that

$$
\{\Xi \in L(E, F):\|\Pi-\Xi\|<\alpha\} \subset \mathfrak{S}(E, F) .
$$

The constant $\alpha$ is called a radius of surjectivity of $\Pi$.

In order to prove our main results, the following two lemmas are also needed.

Lemma 3.7 ([7, Lemma 3.2]). If $(A, \Delta A)$ generates an abstract linear control system on $(X, X)$, then $\left(A, \Delta A, I_{X}\right)$ generates a regular linear system with admissible feedback operator $I_{X}$.

Lemma 3.8. If $(A, P)$ generates an abstract observation system on $(X, X)$, then $\left(A, I_{X}, P\right)$ generates a regular linear system with admissible feedback operator $I_{X}$. 
Proof. For $u \in W_{0, l o c}^{1, p}\left(R^{+}, X\right)=\left\{v \in W_{l o c}^{1, p}\left(R^{+}, X\right): v(0)=0\right\}$, we have that $\Phi_{A, I_{X}}(t) u=\int_{0}^{t} T(t-r) u(r) d r \in D(A), t \geq 0$. Setting $F(\infty) u=P \Phi_{A, I_{X}}(\cdot) u$, by the proof of [6. Proposition 3.3], we obtain that for any $\tau>0, u \in W_{0, l o c}^{1, p}\left(R^{+}, X\right)$,

$$
\begin{aligned}
\|F(\infty) u\|_{L^{p}([0, \tau], X)} & =\left\|P \Phi_{A, I_{X}}(\cdot) u\right\|_{L^{p}([0, \tau], X)} \\
& =\left\|P \int_{0} T(\cdot-s) u(s) d s\right\|_{L^{p}([0, \tau], X)} \\
& \leq \tau^{1 / q}\left\|\Psi_{A, P}(\tau)\right\|\|u\|_{L^{p}([0, \tau], X)},
\end{aligned}
$$

where $q$ satisfies $1 / p+1 / q=1$.

Let $F(\tau)=P_{\tau} F(\infty)$, where $P_{\tau}$ is defined by $\left(P_{\tau} f\right)(t)=f(t)$ when $0 \leq t \leq \tau$ and $\left(P_{\tau} f\right)(t)=0$ when $t>\tau$. It is easy to check that for $u, v \in W_{0, l o c}^{1, p}\left(R^{+}, X\right)$, $F$ satisfies (2.4). Since $W_{0, l o c}^{1, p}\left(R^{+}, X\right)$ is dense in $L_{l o c}^{p}\left(R^{+}, X\right), F(\infty)$ can be extend continuously to $L^{p}\left(R^{+}, X\right)$, and by the same technique as in the proof of 9 , Theorem 3] we obtain that $F$ satisfies (2.4) as well as (2.5) in $L^{p}\left(R^{+}, X\right)$. Thus $\left(A, I_{X}, P\right)$ generates a regular linear system.

It is clear by definition that $\|F(\tau) u\|_{L^{p}([0, \tau], X)}=\|F(\infty) u\|_{L^{p}([0, \tau], X)}$ for any $u \in L^{p}([0, \tau], U)$. Hence, by (3.6) and by the fact that $W_{0, l o c}^{1, p}\left(R^{+}, X\right)$ is dense in $L_{l o c}^{p}\left(R^{+}, X\right)$, we have that $\|F(\tau)\| \leq \tau^{1 / q}\left\|\Psi_{A, P}(\tau)\right\|, \tau>0$. It follows from [26. Proposition 2.3 and Remark 2.4] that $\left\|\Psi_{A, P}(\tau)\right\|$ is bounded on any bounded interval. So, $\|F(\tau)\| \rightarrow 0$ as $\tau \rightarrow 0$. Therefore, $\left(A, I_{X}, P\right)$ generates a regular linear system with admissible feedback operator $I_{X}$.

With the above preparation work, we can prove our main results.

Theorem 3.9. If $(A, B, P)$ generates a regular linear system on $(X, U, X)$, then $(A+P, B)$ generates an abstract linear control system. In addition, if $(A, B)$ is exactly controllable at $\tau>0$, then there exists a constant $\Theta_{0}>0$ such that for any $P$ satisfying $\|P\|_{\mathscr{R}_{A, B}(\tau)}<\Theta_{0}$, the pair $(A+P, B)$ is exactly controllable at $\tau>0$.

Proof. Let $\mathbb{B}:=\left(I_{X}, B\right): X \times U \rightarrow X_{-1}, \mathbb{C}=\left(\begin{array}{c}P \\ 0\end{array}\right): X \rightarrow X \times U$. Then, it follows that $\mathbb{B}$ is admissible for $A$ with the input map $\Phi_{A, \mathbb{B}}=\left(\Phi_{A, I_{X}}, \Phi_{A, B}\right)$, and $\mathbb{C}$ is admissible for $A$ with the output map $\Psi_{A, \mathbb{C}}=\left(\begin{array}{c}\Psi_{A, P} \\ 0\end{array}\right)$. Obviously, $D\left(\mathbb{C}_{\Lambda}^{A}\right)=D\left(P_{\Lambda}^{A}\right)$ and $\mathbb{C}_{\Lambda}^{A}=\left(\begin{array}{c}P_{\Lambda}^{A} \\ 0\end{array}\right)$. Let $F_{1}(\cdot)=\left(\begin{array}{cc}F_{A, I_{X}, P}(\cdot) & F_{A, B, P}(\cdot) \\ 0 & 0\end{array}\right)$. Then, it is easy to verify that $\left(F_{1}(t)\right)_{t \geq 0}$ is a family of bounded linear operators from $L^{p}\left(R^{+}, X \times U\right)$ to $L^{p}\left(R^{+}, X \times U\right)$ and that $\left(T, \Phi_{A, \mathbb{B}}, \Psi_{A, \mathbb{C}}, F_{1}\right)$ satisfies (2.1), (2.3) and (2.4). Moreover, it is easy to see that the transfer function of $F_{1}$ is $G_{1}=\left(\begin{array}{cc}G_{A, I_{X}, P} & G_{A, B, P} \\ 0 & 0\end{array}\right)$, which satisfies the fact that $G_{1}(\lambda)(u, v)^{T} \rightarrow 0$ as $\lambda \rightarrow \infty$ for all $(u, v)^{T} \in X \times U$, by Lemma 3.8 and the assumption that $(A, B, P)$ generates a regular linear system. So, $(A, \mathbb{B}, \mathbb{C})$ generates a regular linear system given by

$$
\left.\Sigma_{A, \mathbb{B}, \mathbb{C}}:=\left(\begin{array}{cc}
T & \left(\Phi_{A, I_{X}}, \Phi_{A, B}\right) \\
\Psi_{A, P} \\
0
\end{array}\right) \quad\left(\begin{array}{cc}
F_{A, I_{X}, P} & F_{A, B, P} \\
0 & 0
\end{array}\right)\right)
$$


with $F_{A, \mathbb{B}, \mathbb{C}}=F_{1}$.

Since by Lemma 3.8 $I_{X}$ is an admissible feedback operator for $\Sigma_{A, I_{X}, P}$, it follows that $I_{X \times U}-F_{A, \mathbb{B}, \mathbb{C}}(\cdot)=\left(\begin{array}{cc}I-F_{A, I_{X}, P}(\cdot) & -F_{A, B, P}(\cdot) \\ 0 & I_{U}\end{array}\right)$ has uniformly bounded inverses, and so $I_{X \times U}$ is an admissible feedback operator for $\sum_{A, \mathbb{B}, \mathbb{C}}$. Thus, in terms of Theorem 2.2, $A^{I_{X \times U}}=\left.\left(A_{-1}+\mathbb{B C}_{\Lambda}\right)\right|_{X}=A+P, B$ is admissible for $A+P$, and

$$
\left(\Phi_{A+P, I_{X}}, \Phi_{A+P, B}\right)=\left(\Phi_{A, I_{X}}, \Phi_{A, B}\right)\left(\begin{array}{cc}
\mathfrak{A} & \mathfrak{A} F_{A, B, P} \\
0 & I_{U}
\end{array}\right),
$$

where $\mathfrak{A}=\left(I_{X}-F_{A, I_{X}, P}\right)^{-1}$. Obviously,

$$
\begin{aligned}
\Phi_{A+P, B} & =\Phi_{A, I_{X}}\left(I_{X}-F_{A, I_{X}, P}\right)^{-1} F_{A, B, P}+\Phi_{A, B} \\
& =\Phi_{A+P, I_{X}} F_{A, B, P}+\Phi_{A, B} .
\end{aligned}
$$

We now prove the robustness of exact controllability. It follows from (3.8) that

$$
\left\|\Phi_{A+P, B}(\tau)-\Phi_{A, B}(\tau)\right\| \leq\left\|\Phi_{A, I_{X}}(\tau)\right\|\left\|\left(I_{X}-F_{A, I_{X}, P}(\tau)\right)^{-1}\right\|\left\|F_{A, B, P}(\tau)\right\|,
$$

and by (3.6) it follows that $\left\|F_{A, I_{X}, P}(\tau)\right\| \leq \tau^{1 / q}\left\|\Psi_{A, I_{X}}(\tau)\right\|$, where $q>1$ satisfies $1 / p+1 / q=1$. Hence, for $\|P\|_{\mathscr{R}_{A, B}(\tau)} \|<\tau^{-1 / q}$, we have

$$
\left\|\Phi_{A+P, B}(\tau)-\Phi_{A, B}(\tau)\right\| \leq \frac{\left\|\Phi_{A, I_{X}}(\tau)\right\|\left\|F_{A, B, P}(\tau)\right\|}{1-\tau^{1 / q}\left\|\Psi_{A, P}(\tau)\right\|} \leq \frac{\left\|\Phi_{A, I_{X}}(\tau)\right\|\|P\|_{\mathscr{R}_{A, B}(\tau)}}{1-\tau^{1 / q}\|P\|_{\mathscr{R}_{A, B}(\tau)}} .
$$

Let $\alpha$ be the radius of surjectivity of $\Phi_{A, B}(\tau)$ and set

$$
\Theta_{0}=\min \left\{\tau^{-1 / q}, \frac{\alpha}{\alpha \tau^{1 / q}+\left\|\Phi_{A, I_{X}}(\tau)\right\|}\right\} .
$$

Then, we have that $\left\|\Phi_{A+P, B}(\tau)-\Phi_{A, B}(\tau)\right\|<\alpha$ if $\|P\|_{\mathscr{R}_{A, B}(\tau)}<\Theta_{0}$. Hence, by Lemma 3.6 it follows that $(A+P, B)$ is exactly controllable at $\tau$ for any $P$ that satisfies $\|P\|_{\mathscr{R}_{A, B}(\tau)}<\Theta_{0}$. The proof is completed.

Remark 3.10. It should be mentioned that when the considered spaces are Hilbert spaces and $p=2$, the admissibility part of the above theorem is contained in [24, Proposition 5.5.2]. In fact, by [29, (7.14)] and Theorem 2.2 it follows that for any $x \in\left(\beta-A_{-1}\right) D\left(\mathbb{C}_{\Lambda}^{A}\right)=\left(\beta-A_{-1}\right) D\left(P_{\Lambda}^{A}\right), J^{A, A+P} x=\left(\beta-(A+P)_{-1}\right)\left(\beta-A_{-1}\right)^{-1} x+$ $\mathbb{B}^{I_{X \times U}} \mathbb{C}_{\Lambda}^{A}\left(\beta-A_{-1}\right) x=\left(\beta-(A+P)_{-1}\right)\left(\beta-A_{-1}\right)^{-1} x+C_{\Lambda}^{A}\left(\beta-A_{-1}\right) x$. This implies that $J^{A, A+P}$ is the same as the corresponding $J$ defined in [24, Proposition 5.52].

Theorem 3.11. If $(A, \Delta A, C)$ generates a regular linear system on $(X, X, Y)$, then $\left(\left.\left(A_{-1}+\Delta A\right)\right|_{X}, C_{\Lambda}^{A}\right)$ generates an abstract linear observation system. In addition, if $(A, C)$ is exactly observable at $\tau>0$, then there exists a constant $k>0$ such that for any $\Delta A$ satisfying $\|\Delta A\|_{\mathscr{W}_{A, C}(\tau)}<k$, the pair $\left(\left.\left(A_{-1}+\Delta A\right)\right|_{X}, C_{\Lambda}^{A}\right)$ is exactly observable at $\tau>0$.

Proof. Consider the operators $\mathscr{B}:=(\Delta A, 0): X \times Y \rightarrow X_{-1}$ and $\mathscr{C}=\left(\begin{array}{c}I_{X} \\ C\end{array}\right):$ $X \rightarrow X \times Y$. It is easy to show that $\mathscr{B}$ is admissible for $A$ with the input mapping $\Phi_{A, \mathscr{B}}=\left(\Phi_{A, \Delta A}, 0\right)$ and $\mathscr{C}$ is admissible for $A$ with the output mapping $\Psi_{A, \mathscr{C}}=\left(\begin{array}{c}\Psi_{A, I_{X}} \\ \Psi_{A, C}\end{array}\right)$. Obviously, $D\left(\mathscr{C}_{\Lambda}^{A}\right)=D\left(C_{\Lambda}^{A}\right)$ and $\mathscr{C}_{\Lambda}^{A}=\left(\begin{array}{c}I_{X} \\ C_{\Lambda}^{A}\end{array}\right)$ in terms of (2.6). Let $F_{2}=\left(\begin{array}{cc}F_{A, \Delta A, I_{X}} & 0 \\ F_{A, \Delta A, C} & 0\end{array}\right)$. Then, it is easy to verify that $\left(F_{2}(t)\right)_{t \geq 0}$ is a 
family of bounded linear operators from $L^{p}\left(R^{+}, X \times Y\right)$ to $L^{p}\left(R^{+}, X \times Y\right)$ and that $\left(T, \Phi_{A, \mathscr{B}}, \Psi_{A, \mathscr{C}}, F_{2}\right)$ satisfies (2.1), (2.3) and (2.4). Moreover, it is easy to see that the transfer function $\left(\begin{array}{cc}G_{A, \Delta A, I_{X}} & 0 \\ G_{A, \Delta A, C} & 0\end{array}\right)$ of $F_{2}$ satisfies $\left(\begin{array}{cc}G_{A, \Delta A, I_{X}} & 0 \\ G_{A, \Delta A, C} & 0\end{array}\right)\left(\begin{array}{l}u \\ v\end{array}\right) \rightarrow$ 0 as $\lambda \rightarrow 0$ for all $(u, v)^{T} \in X \times Y$. Thus $(A, \mathscr{B}, \mathscr{C})$ generates a regular linear system given by

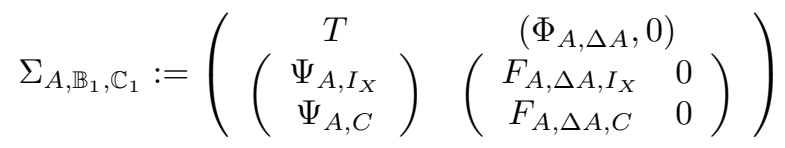

with $F_{2}=F_{A, \mathscr{B}, \mathscr{C}}$.

By Lemma 3.7 it is not difficult to see that $I_{X}$ is an admissible feedback operator for $\left(A, \Delta A, I_{X}\right)$; that is, $I-F_{A, \Delta A, I_{X}}$ has a uniformly bounded inverse, and $I_{X \times Y}-$ $F_{A, \mathscr{B}, \mathscr{C}}=\left(\begin{array}{cc}I-F_{A, \Delta A, I_{X}} & 0 \\ -F_{A, \Delta A, C} & I_{Y}\end{array}\right)$ does as well. Thus, by definition $I_{X \times Y}$ is an admissible feedback operator for $\sum_{A, \mathscr{B}, \mathscr{C}}$. In terms of Theorem 2.2, $A^{I_{X \times Y}}=$ $\left.\left.\left(A_{-1}+\mathscr{B}_{\Lambda}^{A}\right)\right|_{X}=\left\{x \in D\left(\mathscr{C}_{\Lambda}^{A}\right):\left(A_{-1}+\Delta A\right) x \in X\right)\right\}$. This means that $\left(A_{-1}+\right.$ $\left.\mathscr{B} \mathscr{C}_{\Lambda}^{A}\right)\left.\left.\right|_{X} \subset\left(A_{-1}+\Delta A\right)\right|_{X}$. Since both the operators generate $C_{0}$-semigroups, for $\lambda$ large enough we have that $\lambda \in \rho\left(\left.\left(A_{-1}+\mathscr{B}_{\Lambda}^{A}\right)\right|_{X}\right) \cap \rho\left(\left.\left(A_{-1}+\Delta A\right)\right|_{X}\right)$ and

$$
\begin{aligned}
R\left(\lambda,\left.\left(A_{-1}+\Delta A\right)\right|_{X}\right)= & R\left(\lambda,\left.\left(A_{-1}+\Delta A\right)\right|_{X}\right)\left(\lambda-\left.\left(A_{-1}+\Delta A\right)\right|_{X}\right) \\
& \times R\left(\lambda,\left.\left(A_{-1}+\mathscr{B} \mathscr{C}_{\Lambda}^{A}\right)\right|_{X}\right) \\
= & R\left(\lambda,\left.\left(A_{-1}+\mathscr{B} \mathscr{C}_{\Lambda}^{A}\right)\right|_{X}\right),
\end{aligned}
$$

which means $D\left(\left.\left(A_{-1}+\mathscr{B} \mathscr{C}_{\Lambda}^{A}\right)\right|_{X}\right)=D\left(\left.\left(A_{-1}+\Delta A\right)\right|_{X}\right)$. So we get that $A^{I_{X \times Y}}=$ $\left.\left(A_{-1}+\Delta A\right)\right|_{X}$. Hence, by Theorem 2.2 it follows that $C_{\Lambda}^{A^{I} X \times Y}=C_{\Lambda}^{A}$ is admissible for $\left.(A+\Delta A)\right|_{X}$ and

$$
\left(\begin{array}{c}
\Psi_{\left.\left(A_{-1}+\Delta A\right)\right|_{X}, I_{X}} \\
\Psi_{\left.\left(A_{-1}+\Delta A\right)\right|_{X}, C_{\Lambda}^{A}}
\end{array}\right)=\left(\begin{array}{cc}
\left(I_{X}-F_{A, \Delta A, I_{X}}\right)^{-1} & 0 \\
F_{A, \Delta A, C}\left(I_{X}-F_{A, \Delta A, I_{X}}\right)^{-1} & I_{Y}
\end{array}\right)\left(\begin{array}{c}
\Psi_{A, I_{X}} \\
\Psi_{A, C}
\end{array}\right) .
$$

Obviously,

$$
\Psi_{\left.\left(A_{-1}+\Delta A\right)\right|_{X}, C_{\Lambda}^{A}}=F_{A, \Delta A, C}\left(I_{X}-F_{A, \Delta A, I_{X}}\right)^{-1} \Psi_{A, I_{X}}+\Psi_{A, C} .
$$

Below we prove the robustness of exact observability. From the proof of Lemma 3.7 (see [7), we can derive that

$$
\left\|F_{A, \Delta A, I_{X}}\right\| \leq \tau^{1 / p}\left\|\Phi_{A, \Delta A}(\tau)\right\|,
$$

with which we can further prove that, for $\|\Delta A\|_{\mathscr{W}_{A, C}(\tau)}<\tau^{-1 / p}$,

$$
\left\|F_{A, \Delta A, C}(\tau)\left(I_{X}-F_{A, \Delta A, I_{X}}(\tau)\right)^{-1} \Psi_{A, I_{X}}(\tau)\right\| \leq \frac{\left\|\Psi_{A, I_{X}}(\tau)\right\|\|\Delta A\|_{\mathscr{W}_{A, C}(\tau)}}{1-\tau^{1 / p}\|\Delta A\|_{\mathscr{W}_{A, C}(\tau)}}
$$


By the assumption that $(A, C)$ is exactly observable at $\tau$, there exists a constant $k_{0}>0$ such that

$$
\left\|\Psi_{A, C}(\tau)\right\|\|x\| \geq k_{0}\|x\|, x \in X .
$$

Let $0<\alpha_{0}<k_{0}$ and let $k=\min \left\{\tau^{-1 / p}, \frac{k_{0}-\alpha_{0}}{\left(k_{0}-\alpha_{0}\right) \tau^{1 / p}+\Phi_{A, I}(\tau)}\right\}$. Then, we obtain that, when $\|\Delta A\|_{\mathscr{W}_{A, C}(\tau)}<k$,

$$
\begin{aligned}
& \left\|\Psi_{\left.\left(A_{-1}+\Delta A\right)\right|_{X}, C}(\tau) x\right\| \\
\geq & \left\|\Psi_{A, C}(\tau) x\right\|-\left\|F_{A, \Delta A, C}(\tau)\left(I_{X}-F_{A, \Delta A, I_{X}}(\tau)\right)^{-1} \Psi_{A, I_{X}}(\tau) x\right\| \\
\geq & \alpha_{0}\|x\|,
\end{aligned}
$$

for all $x \in X$, which means that $\left(\left.(A+\Delta A)\right|_{X}, C_{\Lambda}^{A}\right)$ is exactly observable at $\tau$. The proof is completed.

Example 3.12. As an illustrative example, we consider the Schrödinger equation with boundary perturbation described by

$$
\begin{cases}w_{t}(x, t)+i \Delta w(x, t)=0, & x \in \Omega, t>0, \\ w(x, t)=0, & x \in \Gamma_{1}, t \geq 0, \\ w(x, t)=L w(x, t), & x \in \Gamma_{0}, t \geq 0, \\ y(x, t)=i \frac{\partial\left(\Delta^{-1} w\right)}{\partial \nu}, & x \in \Gamma_{0}, t \geq 0,\end{cases}
$$

where $\Omega \subset R^{n}, n \geq 2$, is an open bounded region with smooth $C^{3}$-boundary $\partial \Omega=\overline{\Gamma_{0}} \cup \overline{\Gamma_{1}}, \Gamma_{0}$ and $\Gamma_{1}$ are disjoint parts of the boundary relatively open in $\partial \Omega$ and $\operatorname{int}\left(\Gamma_{0}\right) \neq \Omega, \nu$ is the unit normal vector of $\Gamma_{0}$ pointing towards the exterior of $\Omega, u$ is the input function (or control) and $y$ is the output function (or output). Let $H=$ $H^{-1}(\Omega)$ be the state space and let $U=L^{2}\left(\Gamma_{0}\right)$ be the control (input) or observation (output) space. $L$ is a bounded linear operator from $H$ to $U$. The system with $u(t)$ replacing $L w(x, t)$ has been considered by Guo and Shao in [5], where the system (3.12) is cast into the abstract form as (1.1) with well-defined operators $A, B$ and $C$, and it is proved that this system is a regular linear system with feedthrough operator 0 on $(H, U, U)$. By definition, it is not hard to obtain that $(A, B L, C)$ generates a regular linear system on $(H, H, U)$. Moreover, through simple calculation, we can obtain the relation $\|B L\|_{\mathscr{W}_{A, C}(\tau)} \leq\|L\|\|B\|_{\mathscr{W}_{A, C}(\tau)}$. By [17, it can be proved that $D\left(\left.\left(A_{-1}+B L\right)\right|_{H}\right) \subset \mathcal{Z}:=D(A)+\operatorname{Range}\left(\left(\alpha-A_{-1}\right)^{-1} B\right) \subset D\left(C_{\Lambda}^{A}\right)$, where $\alpha \in \rho(A)$ (observe that $\mathcal{Z}$ is independent on $\alpha$ ), and then the system (3.12) can be converted to the observation system $\left(\left.\left(A_{-1}+B L\right)\right|_{H}, C_{\Lambda}^{A}\right)$. According to [12], system (3.12) with $L=0$ is exactly observable at some $\tau>0$. Therefore, by Theorem 3.11. $\left(\left.\left(A_{-1}+B L\right)\right|_{H}, C_{\Lambda}^{A}\right)$, hence (3.12), is an abstract linear observation system and is exactly observable at $\tau$ whenever $\|L\|$ is small enough.

\section{ACKNOWLEDGEMENT}

The authors wish to thank the anonymous reviewers for their helpful and insightful comments and suggestions.

\section{REFERENCES}

[1] S. Boulite, A. Idrissi, L. Maniar, Robustness of controllability under unbounded perturbations, J. Math. Anal. Appl., 304 (2005) 409-421. MR2124672 (2006b:93021)

[2] S. Dolecki, D.L. Russell, A general theory of observation and control, SIAM J. Control and Optim., 15 (1977) 185-220. MR0451141(56:9428) 
[3] K.J. Engel, R. Nagel, One Parameter Semigroups for Linear Evolutional Equations, SpringerVerlag, New York, 2000. MR1721989 (2000i:47075)

[4] B.Z. Guo, G.Q. Xu, Riesz bases and exact controllability of $C_{0}$-groups with one-dimensional input operators, Systems Control Lett., 52 (2004) 221-232. MR2062595 (2005c:93016)

[5] B.Z. Guo, Z.C. Shao, Regularity of a Schrödinger equation with Dirichlet control and collocated observation, Syst. Control Lett., 54 (2005) 1135-1142. MR2170295 (2006d:35208)

[6] S. Hadd, Unbounded perturbations of $C_{0}$-semigroups on Banach spaces and applications, Semigroup Forum, 70 (2005) 451-465. MR2148155 (2006a:47059)

[7] S. Hadd, Exact controllability of infinite dimensional systems persists under small perturbations, J. Evolution Equations, 5 (2005) 545-555. MR2201526 (2006k:93020)

[8] S. Hadd, A. Idrissi, On the admissibility of observation for perturbed $C_{0}$-semigroups on Banach spaces, Systems Control Lett., 55 (2006) 1-7. MR2185598 (2006g:93069)

[9] S. Hadd, A. Idrissi, A. Rhandi, The regular linear systems associated to the shift semigroups and application to control delay systems, Math. Control Signals Systems, 18 (2006) 272-291. MR 2272077 (2007j:34161)

[10] B. Jacob, Exact observability of diagonal systems with a finite-dimensional output operator, Systems Control Lett., 43 (2001) 101-109. MR2007610 (2004f:93017)

[11] A.N. Kolmogorov, S.V. Fomin, Elementi di teoria delle funzioni e di analisi funzionale, Mir, Moscow, 1980.

[12] I. Lasiecka, R. Triggiani, Optimal regularity, exact controllability and uniform stabilization of Shrödinger equations with Dirichlet control, Differential Integral Equations, 5 (1992) 521-535. MR 1157485 (93d:93048)

[13] E.B. Lee, L. Markus, Foundations of Optimal Control Theory, Wiley, New York, 1967. MR0220537 (36:3596)

[14] H. Leiva, Unbounded perturbation of the controllability for evolution equations, J. Math. Anal. Appl., 280 (2003) 1-8. MR1972187(2004b:93015)

[15] J.L. Lions, Contrôlabilité exacte des systèmes distribués, C. R. Acad. Sci. Paris Sér. I Math., 302 (1986) 471-475. MR838402 (87e:93051)

[16] J.L. Lions, Contrôlabilité exacte des systèmes distribués: remarques sur la théorie générale et les applications, in Analysis and optimization of systems (Antibes, 1986), vol. 83 of Lecture Notes in Control and Inform. Sci., Springer, Berlin, 1986, pp. 3-14. MR870385

[17] J. Malinen, O.J. Staffans, Conservative boundary control systems, J. Differential Equations, 231 (2006), 290-312. MR2287888 (2008a:93010)

[18] J.R. Partington, S. Pott, Admissibility and exact observability of observation operators for semigroups, Irish Math. Soc. Bull., 55 (2005) 19-39. MR2185647(2006h:47126)

[19] R. Rebarber, G. Weiss, Necessary conditions for exact controllability with a finite-dimensional input space, Systems Control Lett., 40 (2000) 217-227. MR1827551(2002d:93014)

[20] D.L. Russell, G. Weiss, A general necessary condition for exact observability, SIAM J. Control Optim., 32 (1994) 1-23. MR1255956 (94m:93009)

[21] D. Salamon, Infinite-dimensional linear system with unbounded control and observation: a functional analytic approach, Trans. Amer. Math. Soc., 300 (1987) 383-431. MR876460 (88d:93024)

[22] O.J. Staffans, Well-Posed Linear Systems, Cambridge Univ. Press, Cambridge, UK, 2005. MR2154892 (2006k:93003)

[23] R. Triggiani, Exact boundary controllability on $L_{2}(\Omega) \times H^{-1}(\Omega)$ of the wave equation with Dirichlet boundary control acting on a portion of the boundary $\partial \Omega$, and related problems, Appl. Math. Optim., 18 (1988) 241-277. MR945765 (89i:93011)

[24] M. Tucsnak, G. Weiss, Observation and Control for Operator Semigroups, Birkhäuser Verlag, Basel, 2009. MR2502023 (2010d:93001)

[25] G. Weiss, Admissibility of unbounded control operators, SIAM J. Control Optimization, 27 (1989) 527-545. MR993285 (90c:93060)

[26] G. Weiss, Admissible observation operators for linear semigroups, Israel J. Mathematics, 65 (1989) 17-43. MR994732 (90g:47082)

[27] G. Weiss, The representation of regular linear systems on Hilbert spaces, in: Kappel, F., Kunisch, K., Schappacher, W. (eds.), Control and estimation of distributed parameter systems (Proceedings Vorau, 1988), Birkhäuser, pp. 401-416. MR.1033074 (91d:93026)

[28] G. Weiss, Transfer functions of regular linear systems. Part I. Characterizations of regularity, Trans. Amer. Math. Soc., 342 (1994) 827-854. MR1179402 (94f:93074) 
[29] G. Weiss, Regular linear systems with feedback, Math. Control Signals Systems, 7 (1994) 23-57. MR1359020 (96i:93046)

[30] G.Q. Xu, C. Liu, S.P. Yung, Necessary conditions for the exact observability of systems on Hilbert space, Systems Control Lett., 57 (3) (2008) 222-227. MR.2386920 (2008j:93028)

Department of Mathematics, Xi'an Jiaotong University, Xi'an 710049, People's Republic OF ChinA

E-mail address: mzhd1516@gmail.com

Department of Mathematics, Xi'an Jiaotong University, Xi'an 710049, People's Republic of ChinA

E-mail address: jgpeng@mail.xjtu.edu.cn 